\title{
Fehldeutungen der Physik und Philosophie in der Alternativmedizin*
}

Martin Lambeck

\footnotetext{
* Festrede gehalten am 4. September 2008 anlässlich des Symposiums zum

70. Geburtstag von Prof. Dr. med. Brunello Wüthrich.
}

\section{1 http://de.wikipedia.org/wiki/} Large_Hadron_Collider

Korrespondenz:

Prof. Dr. Martin Lambeck

Technische Universität Berlin

Institut für Optik und atomare Physik

Sekretariat ER 1-1

Strasse des 17. Juni 135

D-10623 Berlin

martinlambeck@web.de

www.gwup.org/lambeck
Wie kommt man zum Nobelpreis?

Frage: Wie kommt man zum Nobelpreis? Hierfür sehe ich drei Möglichkeiten:

Die erste Möglichkeit ist charakterisiert durch Namen wie Röntgen, Planck, Einstein, Heisenberg usw. Also: der geniale Einzelforscher.

Ein Beispiel für die zweite Möglichkeit sehen wir jetzt in der Schweiz. In Genf entsteht der «Large Hadron Collider». Wir sehen einen kleinen Teil des Experiments - hier ist ein Mensch. Das Experiment wird 27 Kilometer lang und über 3 Milliarden Euro kosten [1]. Das nenne ich: «Nobelpreis mit Gewalt».

Die dritte Möglichkeit erkläre ich Ihnen jetzt:

\section{Axiome der Schulmedizin}

Die naturwissenschaftlich begründete Medizin erklärt die Wirkung von Medikamenten, Allergenen, Giften usw. dadurch, dass diese Stoffe im Körper auf der molekularen Ebene wirken. Man spricht vom «Andocken an einen Rezeptor». Ein Medikament kann nur wirken, wenn seine Moleküle sehr nahe an die Moleküle des Körpers herankommen, so dass die Elektronenwellen, also die Materiewellen beider Moleküle, sich überlappen und so eine chemische Bindung beider Moleküle nach dem Schloss-Schlüssel-Prinzip bewirken. Derartige Abstände liegen in der Grössenordnung von 0,1 Nanometern, also etwa einem Atomdurchmesser. Die Verbindung der beiden Moleküle erfolgt vergleichsweise so, wie wenn wir uns die Hand geben und so ein «menschliches Molekül» bilden.

\section{Materie-Nah-Medizin}

Zusammengefasst gibt es zwei Aussagen: 1. Die Medikamente müssen als Materie vorhanden sein. 2. Sie wirken nur über Entfernungen von etwa 0,1 Nanometer. Diese beiden Aussagen haben nahezu den Rang von Axiomen und werden in der Medizin als so selbstverständlich angesehen, dass sie kaum noch erwähnt und schon gar nicht in Frage gestellt werden. Daher bezeichne ich diese Richtung der Medizin als «Materie-Nah-Medizin». Die Naturheilkunde, so wie ich sie verstehe, ist ein Teil der naturwissenschaftlichen Medizin.

Machen wir uns klar, dass es hier um die Fundamente der Naturwissenschaft geht: Wenn es gelingen sollte, ein wirksames medizinisches Ver- fahren zu finden, das einem der beiden Axiome oder beiden widerspricht, dann wäre das für die Physik so wie die Entdeckung der Röntgenstrahlen und der Kernspaltung, für die Medizin wie die Entdeckungen von Semmelweis und Koch. Das nenne ich Phänomene 1. Klasse. Wenn irgendwann einmal der vielstrapazierte Begriff «Paradigmenwechsel» angebracht sein sollte, dann hier!

\section{Hufelandgesellschaft}

\section{Schwingungs-Nah-Medizin}

Tatsächlich gibt es heute Gebiete der «alternativen Medizin», in denen eines der beiden Axiome oder beide nicht mehr gelten sollen. Dazu gehören die Homöopathie und die anthroposophisch erweiterte Heilkunst. Die Herstellung der zugehörigen Medikamente ist im Homöopathischen Arzneibuch (HAB) geregelt. Danach werden Medikamente durch «Potenzieren» (stufenweises Verdünnen mit dazwischengeschaltetem Schütteln) hergestellt. Dabei darf bzw. soll die Potenzierung so weit getrieben werden, dass das fertige Medikament kein einziges Molekül der Ausgangssubstanz mehr enthält (Hochpotenz). Damit ist das erste Axiom verletzt, denn es ist keine Materie der Ausgangsubstanz mehr vorhanden. Statt dessen befindet sich darin eine - wie Hahnemann in der Sprache seiner Zeit sagte - «geistartige Kraft». Heute spricht man von «Information» oder «Schwingung». Wir haben hier also eine «Schwingungs-Nah-Medizin».

Grosser Beliebtheit erfreut sich ein Verfahren, das als «Bioresonanz» bezeichnet wird. Hierbei wird angenommen, dass der Mensch Schwingungen aussendet, die für seine Krankheit spezifisch sind. Diese Schwingungen werden vom Bioresonanzgerät aufgenommen, verstärkt, in ihrer Phase umgekehrt und dem Patienten wieder zugeleitet. Hierdurch sollen die ursprünglichen Krankheitsschwingungen «gelöscht» und der Patient geheilt werden.

Wenn ich den Abstand der chemischen Bindung an den Rezeptor mit der Handschlagentfernung vergleiche, dann entspricht die Entfernung des Patienten vom Bioresonanzgerät der Entfernung Erde-Mond. Daher spreche ich hier von Fernmedizin. Somit ist das zweite Axiom verletzt. 
Insgesamt ergibt sich so das Bild einer «Schwingungs-Fern-Medizin». Damit lautet die Frage: Können Materiewellen durch elektromagnetische Wellen ersetzt werden?

\section{Die Dachgesellschaft} und ihre Gebührenordnung

Mehrere medizinische Gesellschaften, deren Verfahren zur Physik in einem erklärungsfordernden Spannungszustand stehen, haben sich zu einem Dachverband zusammengeschlossen, der Hufelandgesellschaft. Der Vorstand der Hufelandgesellschaft und Prof. Dr. Manfred Rimpler haben ein Leistungsverzeichnis herausgegeben, das als Gebührenordnung dient. Es wird von 20000 deutschen Ärzten und mehreren grossen Versicherungsgesellschaften verwendet. Hier sind nach meiner sehr vorsichtigen Schätzung 14 Nobelpreise drin [2].

Ich kann jetzt nicht auf alle Verfahren und Gesellschaften eingehen, sondern konzentriere mich auf die Bioresonanz sowie die Gesellschaften in Deutschland und der Schweiz.

\section{Quantenphysikalische Hufeland- phänomene}

\section{Schwingungsozean}

Für die Bioresonanz gibt die Hufelandgesellschaft folgende physikalische Begründung:

Hufeland-Leistungsverzeichnis der Besonderen Therapieeinrichtungen. Bearbeitet von Prof. Dr. Manfred Rimpler. 4. Auflage. Stuttgart: Karl F. Haug; 2005.
«Die Bioelektronische Systemdiagnostik und -theraphysik. Danach besteht alles im Universum aus elekpie basiert auf den Erkenntnissen der Quanten- tromagnetischer Schwingung, auch der Mensch. Die Masse macht dabei den unbedeutenden Teil von etwa $0,001 \%$ aus. Lebende Systeme sind in der Lage, aus dem uns umgebenden elektromagnetischen Ozean sinnvolle, den Ordnungsgrad steigernde Signale herauszufiltern und zu verarbeiten.»

Auf diese Weise wird aus der Quantenphysik eine Verbindung des Menschen mit dem Universum, also eine ganzheitliche Weltsicht, abgeleitet. Rimpler gibt einen Prozentsatz an. Danach hat die mit mir verbundene elektromagnetische Schwingung eine Masse von 7500 Tonnen. Davon habe ich noch nie etwas gemerkt.

\section{Schwingungs-Fern-Medizin = Bioenergetische Medizin}

\section{Bioresonanz-Molekularsaugkreis}

Die medizinische Wirksamkeit der Bioresonanz wird von der Hufelandgesellschaft folgendermassen begründet:

«Die Weiterentwicklung dieses Konzeptes ermöglichte es, durch Nutzung des Resonanzprinzips mit Hilfe eines besonderen biologischen Filters (Molekularsaugkreis) die körpergünstigen physiologischen Schwingungen $(\mathrm{H})$ von den körperungünstigen pathologischen Schwingungen (D) im Gerät elektronisch zu trennen und diese getrennten Informationsmuster bezüglich der Verstärkungen und der Frequenzbereiche unterschiedlich zu bearbeiten.» [H = Harmonisch; D = Disharmonisch; M.L. $]$

Der Begriff «Saugkreis» ist mir seit meiner Schulzeit als Radiobastler bekannt. Von der Fähigkeit,

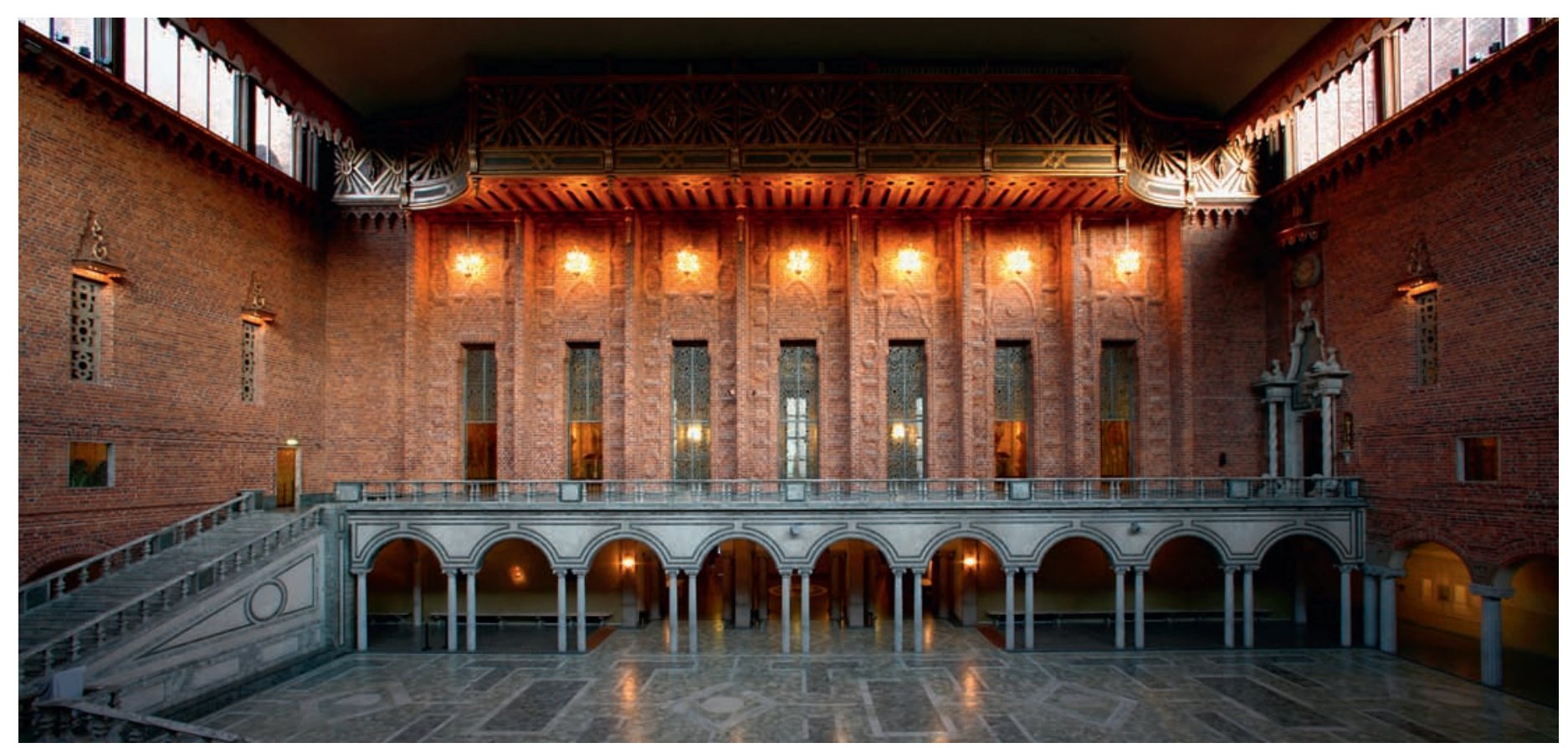


gesundheitsschädliche von gesundheitsfördernden Schwingungen zu unterscheiden, habe ich noch nie etwas gehört. Eine solche Fähigkeit wäre ein Phänomen erster Klasse, vergleichbar der Entdeckung der Röntgenstrahlen und der Kernspaltung.

\section{Bioresonanz SABIT}

In der Schweiz gibt es die Schweizerische Ärztegesellschaft für Biophysikalische Informationstherapie (SABIT). Allein im Kanton Zürich arbeiten 16 Mitglieder der SABIT, 14 Ärzte und 2 Zahnärzte. Die SABIT erklärt [3]:

«Bei der biophysikalischen Informationstherapie (BIT) handelt es sich um ein energetisches Therapieverfahren, das auf den Grundlagen der Quantenphysik basiert und dabei den Informationsgehalt elektromagnetischer Schwingungen benützt. Krankheit kann entstehen durch fehlerhafte Informationsübertragung, welche dann zu Fehlsteuerung biochemischer Reaktionen führt.» [Info für Ärzte/Fertigkeitsprogramm].

«Die persönlichen Schwingungen des Patienten werden von bestimmten Arealen der Körperoberfläche mittels Elektroden abgegriffen und ins Therapiegerät geleitet. Dort werden sie in individuell ausgetesteter Weise verändert und dem Körper danach als Therapieschwingungen auf bestimmte Stellen zurückgegeben. [...] Diese Therapieform wird hauptsächlich gebraucht bei der Behandlung von Infektionen, zur Giftausleitung oder Allergietherapie.» [Info für Patienten/BIT Allgemein]

Sehen wir uns an, welchen Schatz die SABIT hiermit hütet: Der Patient sendet krankheitsspezifische elektromagnetische Signale aus. Das bringt einen Nobelpreis für Medizin. Diese Signale, die in der Medizin bisher nicht bemerkt wurden, weshalb sie in der Literatur als «ultraschwach» bezeichnet werden, werden vom Eingangsverstärker des Bioresonanzgerätes verstärkt. Das bringt einen Nobelpreis für Physik. Danach werden sie vom Molekularsaugkreis entsprechend ihrer Gesundheitsfreundlichkeit getrennt. Noch einer für Physik. Die Ausgangsschwingungen des Gerätes bewirken Heilung des Patienten - noch einer für Medizin. Die Eingangsbecher nehmen die Schwingungen von Allergenen wie Katzenhaaren oder Birkenpollen auf - noch mal einer für Physik. Insgesamt ist also schon dieses eine Bild der Wegweiser zu fünf Nobelpreisen - ein echter Service der SABIT!

\section{Konsequenzen für Wissenschaft und Versicherungen}

Somit ergibt sich die Alternative: Entweder funktioniert dieses Gerät. Dann ist das eine mehrfachnobelpreisfähige Revolution von Physik, Medizin und Elektrotechnik. Oder es funktioniert nicht. Dann vermittelt das Hufelandleistungsverzeichnis die Empfehlung von Honoraren für eine Leistung, die gar nicht erbracht werden kann.

\section{Erklärung durch Holismus}

Während Rimpler nur den speziellen physikalischen Mechanismus der elektromagnetischen Schwingungen heranzieht, gibt es einen viel umfassenderen Ansatz, der auch zu tiefgreifenden philosophischen Konsequenzen führt. Den Begriff «ganzheitlich» haben wir schon gehört. Dieser Ansatz wird als «Ganzheitlichkeit» oder vom griechischen Wort «holos», d. h. "ganz»als «Holismus» bezeichnet.

Zur Vorgeschichte: Es gibt einen äusserst interessanten Quanteneffekt, der auf ein Gedankenexperiment von Einstein, Podolsky und Rosen zurückgeht, weshalb er als EPR-Effekt bezeichnet wird [4]. Wenn ein Atom gleichzeitig zwei Photonen emittiert, verhalten sich diese beiden Photonen wie Zwillinge. Die Physiker sagen: Die beiden Photonen sind «verschränkt». In alleräusserster Vereinfachung gesagt: Wenn man eins dieser verschränkten Photonen dreht, muss sich auch sein Zwilling drehen, selbst wenn er sich schon auf dem Jupiter befindet, wohin das Licht eine Stunde braucht.

\section{Capra}

Diese «Verschränkung» der Quantenzwillinge ist die Grundlage für Fritjof Capras Bestseller «Der kosmische Reigen. Physik und östliche Mystik ein zeitgemässes Weltbild», dessen Kernsatz lautet: Jeder Teil des Universums ist zugleich das Ganze, und das Ganze ist zugleich jeder Teil alles Sein durchdringt sich gegenseitig [5].

\section{Kafatos/Nadeau}

Dieser Gedanke wurde von Kafatos und Nadeau weiterentwickelt zu ihrem Buch «The Conscious Universe - Part and Whole in Modern Physical Theory» [6]. Bereits der Umschlag zeigt programmatisch: Das Gehirn als Symbol für das menschliche Bewusstsein und ein Sternenbild als Symbol für das Universum. Darunter die Versuchsanordnung, mit der zum ersten Mal die EPR-Verschränkung von Photonen nachgewiesen wurde. Das Bild sagt also: Durch den quantenphysikalischen Verschränkungseffekt, EPR, ist das menschliche Bewusstsein mit dem ganzen Universum verbunden. Somit ist das Universum ganzheitlich - «holistisch». Hier wird also das holistische Weltbild auf einem Buchumschlag mit einem Blick erkennbar.

\section{Dahlke}

Der bekannteste deutsche Esoteriker, Dr. Rüdiger Dahlke, verwendet ebenfalls das EPR-Phänomen, um zu begründen, «dass in diesem Universum 
alles mit allem zusammenhängt». Er gelangt somit zum Prinzip der Esoterik «Wie oben, so unten» und zum Satz «Der Mensch und die Welt sind eins». Daher wird schon im Umschlagbild der Mensch vor der Erdkugel dargestellt [7].

\section{Hanzl}

Dieses holistische Weltbild wird nun auch zur Erklärung der Schwingungs-Fern-Medizin, wie der Bioresonanz herangezogen.

Der deutsche Arzt Dr. Günther S. Hanzl (www.regulations-medizin.com) arbeitet im Vorstand der Internationalen Medizinischen Gesellschaft für Elektroakupunktur nach Voll (IMGEAV) und ist damit auch prominentes Mitglied der Hufelandgesellschaft. Ausserdem ist er Schriftleiter der zugehörigen Zeitschrift «Regulationsmedizin», folglich als der führende Kopf der Schwingungs-Fern-Medizin anzusehen. Hanzl hat völlig richtig erkannt, dass die Bioresonanz sowie ähnliche Verfahren eine Revolution von Physik und Medizin bedeuten und daher ein Buch geschrieben mit dem programmatischen Titel «Das neue medizinische Paradigma» [4].

Hanzl versucht keineswegs, diese Revolution mittels einer neuen, bislang unbekannten Wissenschaft zu begründen, sondern er unternimmt es, die Revolution auf die schon bekannte Physik, nämlich das EPR-Phänomen, zu stützen. Hieraus leitet Hanzl eine «nichtlokale» Verbindung der Gegenstände ab, so dass auch ein krankes Organ des Patienten auf das Bioresonanzgerät wirken kann.

Nicht-lokale Wechselwirkungen wirken augenblicklich und ohne Verzögerung, da ja zwischen den Objekten nicht-lokaler Verknüpfung weder Materie noch Raum eine Rolle spielen kann. Sie sind - bei beliebiger Distanz - in der nichtlokalen Realität ungetrennt! (86).

Die universelle vernetzte Kausalität [...] bewirkt, [...] dass alles mit allem in Beziehung steht (135).

Das ganze Universum ist ein Hologramm (137). [4]

Capra und Nachfolger kommen durch die Anwendung der Quantenphysik und EPR zum holistischen Weltbild und verwenden dieses in Philosophie und Medizin. Dass ich dieses Weltbild für falsch halte, habe ich schon früher beschrieben [8]. Jetzt werde ich aus der Quantenphysik und EPR ein nichtholistisches Weltbild entwickeln. Zur Erklärung der erstaunlichen Tatsache, dass ich aus derselben Physik den entgegengesetzten Schluss ziehe, habe ich das nachfolgende Beispiel gebildet.

\section{Kerzenvergleich}

In einer alten Burg soll demnächst ein Fest stattfinden. Zur Vorbereitung kommt der Koch mit zwei Jungen in die Küche. Dort zündet er gleichzeitig zwei gleich grosse Kerzen an. Mit der einen schickt er einen Jungen in den Bierkeller zum Prüfen der Vorräte, den anderen in den Weinkeller. Also werden die Kerzen mit gleicher Geschwindigkeit brennen und gleichzeitig erlöschen. Das tun sie aufgrund ihrer eigenen inneren Eigenschaften. Die Fehldeutung der EPR-Verschränkung besteht in der Annahme, der Bierkeller habe auf den Weinkeller gewirkt. Capra und andere Fehldeuter der Quantenphysik verwechseln die Eigenschaften der verschränkten Quanten mit den Eigenschaften der Umgebung.

\section{Dekohärenz}

Mein Beispiel trägt noch weiter: Die beiden Kerzen sind aufgrund ihrer Eigenschaften zunächst zusammenhängend, physikalisch ausgedrückt «kohärent». Wenn im Bierkeller Zugluft herrscht, wird diese Kerze flackern und früher erlöschen. Die Umwelt zerstört die Gleichheit der Kerzen, sie sind jetzt «dekohärent». Diese «Dekohärenz» bewirkt, dass eine Kerze einige Minuten früher erlischt. Wie schnell geht diese Zerstörung im Quantenbereich?

Manche Menschen haben eine Allergie gegen Birkenpollen. Eine solche Birkenpolle wird allein dadurch, dass sie in der Luft fliegt, dekohärent, und zwar nach $10^{-18}$ Sekunden (Dekohärenz). $10^{-18}$ Sekunden heisst nach menschlichen Massstäben: sofort. Eine Birkenpolle - und erst recht ein so grosses Gerät wie ein Bioresonanzgerät zeigt also keine Quanteneffekte, so dass das holistische Weltbild und alle Erklärungen von Capra bis Hanzl zusammenbrechen.

\section{Aufruf}

Wenn das, was die von der Hufelandgesellschaft und der SABIT vertretenen Ärzte jeden Tag tun, richtig ist, ist die Physik falsch oder zumindest grob unvollständig. Das führt mich zurück zur Ausgangsfrage: Wie kommt man zum Nobelpreis?

Wir brauchen kein Experiment, das 27 Kilometer lang ist und 3 Milliarden Euro kostet. Die Geräte, mit denen die Hufeland- und SABIT-Phänomene produziert werden, sind bereits in Tausenden Arztpraxen vorhanden. Die Ärzte haben jahrelange Erfahrung in ihrem Gebrauch.

Also rufe ich Tausende deutsche und Schweizer Ärzte auf: Holen Sie die Nobelpreise, falsifizieren Sie die Physik. Wohlan! 